\title{
A numerical study of flow past an transversely oscillating wavy cylinder at $R e=5000$
}

\author{
Kai Zhang, ${ }^{\mathrm{a}, \mathrm{b}}$, Hiroshi Katsuchi ${ }^{\mathrm{b}, *}$, Dai Zhou ${ }^{\mathrm{a}, \mathrm{c}, \mathrm{d}}$, Hitoshi Yamada ${ }^{\mathrm{b}}$ \\ ${ }^{a}$ School of Naval Architecture, Ocean and Civil Engineering, Shanghai Jiao Tong University, Shanghai, 200240, China \\ ${ }^{b}$ Department of Civil Engineering, Graduate School of Urban Innovation, Yokohama National University, Yokohama, \\ 2408501, Japan \\ ${ }^{c}$ Collaborative Innovation Center for Advanced Ship and Deep-Sea Exploration (CISSE), Shanghai, 200240, China \\ ${ }^{d}$ State Key Laboratory of Ocean Engineering, Shanghai Jiao Tong University, No. 800, Dongchuan Road, Shanghai, \\ 200240, China
}

\begin{abstract}
In order to shed some light upon the mechanism for the free vibrations of the wavy cylinder reported previously [18, we numerically investigate the effect of external forcing on the flow around a wavy cylinder at $R e=5000$. The forcing takes the form of sinusoidal transverse motion with the amplitude fixed at $A_{e} / D_{m}=0.2$ and the frequency spanning from $f_{e}=0.1$ to 0.3 . Even though the wavy cylinder is optimally designed to annihilate the Kármán vortex shedding in the fixed configuration, the Strouhal frequency is found to resurrect in the forced vibration cases, leading to very similar responses between the normal and wavy cylinders, especially at high forcing frequencies. By sectional analysis it is revealed that the sectional distribution of the force coefficients undergoes significant changes with the increase of the forcing frequency. Statistical tools such as the correlation and coherence reveals further spanwise features of the force coefficients. In addition, the instantaneous three-dimensional vortical structures are visualized by the spanwise voriticity isosurfaces to collaborate the previous discussions. The cessation of the flow control efficacy in the forced vibration is explained by the recession of the mean streamwise vorticity contours. The results presented in the work imply that the vortex-induced vibration of the wavy cylinder might be ascribed to the typical wake-structure interaction as the normal cylinder, rather than merely the destabilization of the unstable structure mode as we have surmise earlier.
\end{abstract}

Keywords: Wavy cylinders; forced vibration; lock-in; correlation; coherence; VIV

\section{Introduction}

The flow around bluff bodies has been a celebrated topic of research not only owing to its common occurrence in engineering applications, but also the profound significance in the fundamental fluid mechanics. More often than not, efforts are made to suppress the vortices behind the bluff structures 5 in order to reduce the detrimental drag and fluctuating lift forces exerted on the bodies. Recently, the 3D forcing technique [1, which applies span-wise varying controls for the nominally two dimensional bluff bodies, has been recognized as an effective and efficient way of regulating the wake flow. Typical examples pertaining to this category includes the distributed blowing and suction [2, hemisphere bumps [3], wavy stagnation surfaces [4, etc.

In engineering applications such as bridge cables and offshore pipelines, the flow could attack the structures from all possible angles, requiring the control approaches to be omni-directional. One particular kind of the 3D forcing device, which meets such requirement, is the wavy circular cylinder, i.e., straight axis with sinusoidally varying diameter. The cross flow of the such cylinders has been subjected to intensive researches over the last two decades [5-13]. The waviness has been shown to 15 generate significant span-wise pressure gradient on the cylinder surface, yielding an uneven distribution

*Corresponding author. Email Address: katsuchi@ynu.ac.jp 
of the sectional force coefficients. Integrating the forces along the axial direction results in significantly smaller total drag and lift forces compared with the normal cylinder. The flow control efficacy of the wavy cylinder depends highly on the shape parameters, i.e., the geometric amplitude and wavelength. Two optimal wavelengths at $\lambda / D_{m} \approx 2$ and 6 have been found to be particularly effective in the force mitigation. Provided with suitable geometric amplitude, the wavy cylinder could even completely annihilates the Kármán vortex shedding behind it. As has been explained by [9, 13, the mechanism of the flow control lies in the formation of the periodic streamwise vortices, which stabilize the free shear layers and inhibit them from rolling up to forming the span-wise Kármán vortices. From a more theoretical perspective, 14] revealed in the light of linear stability analysis that the span-wise waviness in the two-dimensional base flow wake results in stabilization of the absolute instability. Apart from the cylindrical structures, the spanwise waviness has also been found to exist in the flippers of the humpback whales [15], inspired by which many innovations such as the wavy wings and wind turbines has been proposed [16, 17. In view of the above, the wavy cylinder presents a promising passive flow control device that has the potential to be used in various engineering applications.

Given the satisfactory flow control efficacy exhibited by the stationary wavy cylinders, we numerically investigated the flow around a flexibly mounted one at $R e=5000$, expecting that the vortexinduced vibration could also be effectively mitigated [18. The wavy cylinder was optimally designed so that it completely suppresses the Kármán instability at the studied Reynolds number. It was to our disappointment to find that the free vibration response against the reduced velocity largely resembles that of the normal cylinder. The maximum vibration amplitude is only $15 \%$ smaller compared with the normal cylinder, while for the same shape in the static configuration the lift force reduction is around $90 \%$. More interestingly, even in the absence of the primary vortex shedding frequency, the frequency response from the oscillating wavy cylinder also exhibits the typical lock-in phenomenon at a similar range of reduced velocity with the normal cylinder. These findings attested to the statement in controlling the vibrations.

In order to shed more light upon the glaring differences exhibited by the wavy cylinder at fixed and flexibly-supported configurations, the current work embark upon a curiosity study on the effect of external sinusoidal oscillation on the cross flow of the wavy cylinders. Such a problem has been hereafter) by both experimental [20-26] and numerical 27-31 methods, as a complementary approach to understand the more complex fluid-structure interaction problems. For a static cylinder placed in the uniform stream, the von Kármán vortex shedding with the fixed frequency $f_{v}$ prevails the wake flow at the subcritical Reynolds numbers. Such shedding process is disturbed and complicated as the 50 forced motion with amplitude $A_{e}$ and frequency $f_{e}$ intervenes. This is clearly manifested by the various vortex shedding modes at their respective combinations of $A_{e}$ and $f_{e}$ as is tabulated in [32. For the forcing frequencies significantly different from the Strouhal frequency, the spectrum of the flow variables (near wake velocity, lift coefficient, etc.) would reveal two distinctive peaks with one located at $f_{e}$ and the other in the vicinity of $f_{v}$. As the forcing frequency approaches the natural vortex shedding the submission of the shedding frequency to the forcing, could be expected. The extent of the lock-in region is found to be positively dependent on the prescribed oscillation amplitude. In addition, the frequency synchronization is accompanied with many other features such as the magnification of the drag and lift forces, the sudden change of phase between the lift and displacement, change of vortex patterns, etc. In general, the forced vibration share many features, frequency lock-in in particular, with the free vibrations 33 35. This has led to some successful predictions of the VIV by the forced oscillation tests [26, 31, 36]. It should also be noted that the frequency synchronization phenomenon is ubiquitous to many other kinds of forcing such as the oscillatory jet control to the cylinder wake [37, unsteady blowing-suction to post-stall flow control of an airfoil [38, the localized periodic heating to the shear layer modification [39, etc.

In view of the above, the change of state from desynchronization to the lock-in in the forced vibration of a circular cylinder is essentially owing to the evolution of the complicated entrainment of the vortex shedding process to the prescribed forcing. On the other hand, we have shown in our previous work [18] that the optimally designed wavy cylinder could completely eliminate the Kármán 
vortex shedding even in the turbulent regime. This renders the current research, the forced vibration of a wavy cylinder, very interesting, since such a cylinder is devoid of the Strouhal frequency in the fixed configuration. Some hints have been revealed in our previous 18 that the concealed Strouhal frequency would resurface in the dynamic case and play its part in determining the free vibration responses. It is believed that a thorough investigation by the forced vibration would bring more insights to this problem. With the motivation described above, the rest of the paper is organized as follows. In the section to come, a detailed description of the case setup is presented. This is followed by an introduction of the governing equations and the numerical schemes in section 3 . Discussion of the results are divided into three major parts in the next section. Firstly, the wavy cylinder is viewed as whole and its global drag and lift coefficients are compared with that of the normal cylinder. In the second part, sectional analysis of the wavy cylinder is presented. The sectional distribution of the force coefficients, as well as the spanwise correlation and coherence will be covered. To supplement the above discussions, the vortex shedding modes at various forcing frequencies are presented in the third part. Conclusions, together with some prospects for future research, are presented in the last section.

\section{Description of the problem}

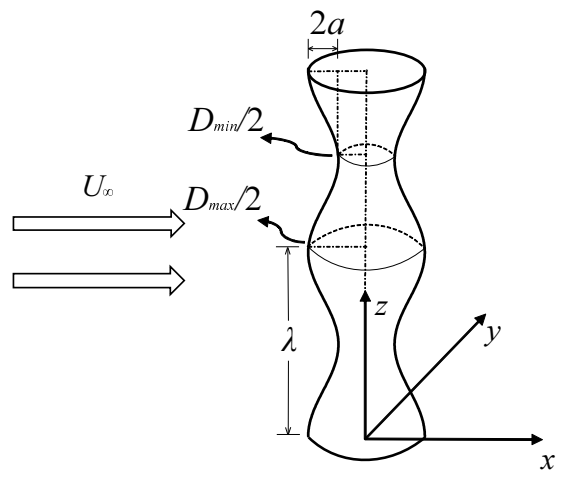

(a) Shape and coordinate

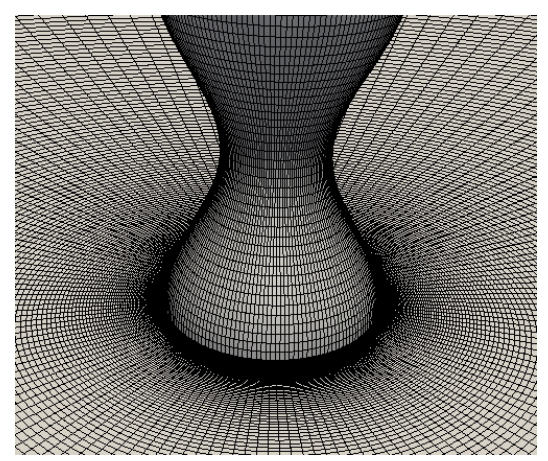

(b) Overview of the mesh

A schematic of the geometry of interest with coordinate system is presented in Fig. 1(a). The diameter of the wavy cylinder varies sinusoidally along its span-wise direction by the following equation:

$$
D_{z}=D_{m}+2 a \cos (2 \pi z / \lambda),
$$

where $D_{z}$ denotes the local diameter of the wavy cylinder and $D_{m}=\left(D_{\min }+D_{\max }\right) / 2$ is the averaged diameter which is used in the calculation of Reynolds number as well as the averaged force coefficients. $\lambda$ is the geometric wavelength in the span-wise direction and the geometric amplitude $a$ is the maximum deviation of local radius from the average radius $D_{m} / 2$. The axial locations at which the local diameter maximizes $\left(D_{\max }\right)$ or minimizes $\left(D_{\min }\right)$ are referred to as 'node' and 'saddle', respectively. In the current paper, we assign $\lambda / D_{m}=2$ and $a / D_{m}=0.175$. A total length of cylinder $H=4 D_{m}$, which encompass two wavelengths, has been adopted. This particular wavy shape has been proven to completely suppress the Kármán vortex shedding in the fixed configuration at Reynolds number of $5000[18$.

Placed in the uniform streamwise flow, the cylinder, which is aligned with the $z$ direction, is forced to vibrate rigidly in the transverse $(y)$ direction according to the following equation:

$$
Y(t)=A_{e} \sin \left(2 \pi f_{e} t\right),
$$

here, the normalized oscillation amplitude $A_{e} / D_{m}$ is fixed at 0.2 and $f_{e} D_{m} / U$ is varied from 0.1 to 0.3 with an interval of 0.01 . Note that in the following discussion, we will simply use $A_{e}$ and $f_{e}$ to denote the non-dimensional quantities. For the purpose of comparison, the same simulations are also conducted for the normal circular cylinder. For the fixed normal cylinder the Strouhal frequency is around $f_{v} \approx 0.21$. Thus, the selected excitation frequency spans from around $0.5 f_{v}$ to $1.5 f_{v}$. 


\section{Governing equations, boundary conditions and mesh}

The Navier-Stokes equations are spatially filtered to yield the governing equations of Large Eddy

Simulation (LES), which, in the Arbitrary-Lagrangian-Eulerian (ALE) frame, read as follows:

$$
\begin{gathered}
\frac{\partial \bar{u}_{i}}{\partial x_{i}}=0 \\
\frac{\partial \bar{u}_{i}}{\partial t}+\left(\bar{u}_{j}-\tilde{u}_{j}\right) \frac{\partial \bar{u}_{i}}{\partial x_{j}}=-\frac{\partial \bar{p}}{\partial x_{i}}+\frac{\partial}{\partial x_{j}}\left[\nu\left(\frac{\partial \bar{u}_{i}}{\partial x_{j}}+\frac{\partial \bar{u}_{j}}{\partial x_{i}}\right)+\tau_{i j}\right],
\end{gathered}
$$

where $\left(x_{1}, x_{2}, x_{3}\right)=(x, y, z)$ are the Cartesian coordinates, $\bar{u}_{i}$ is the filtered velocity tensor and $\bar{p}$ is the filtered pressure, in which the constant fluid density $\rho$ has been incorporated. $\tilde{u}_{i}$ is the velocity component of mesh moving in the $x_{i}$ direction. $\tau_{i j}=\overline{u_{i} u_{j}}-\bar{u}_{i} \bar{u}_{j}$ is the sub-grid scale (SGS) stress that requires extra modeling. The SGS stress is expressed in a wavy reminiscent of the Boussinesq hypothesis with the introduciton of a turbulent eddy viscosity $\nu_{t}$ :

$$
\tau_{i j}-\frac{2}{3} k_{t} \delta_{i j}=-2 \nu_{t} \bar{S}_{i j}
$$

in which $k_{t}=\tau_{k k} / 2$ is the SGS turbulent kinetic energy and $\bar{S}_{i j}=\frac{1}{2}\left(\frac{\partial \bar{u}_{i}}{\partial x_{j}}+\frac{\partial \bar{u}_{j}}{\partial x_{i}}\right)$ is the strain rate tensor calculated directly from the resolved scales. In the current paper, the dynamic $k$-equation model is adopted to solve for the $k_{t}$ and $\nu_{t}$, i.e.,

$$
\begin{aligned}
\frac{\partial k_{t}}{\partial t}+\frac{\partial}{\partial x_{j}}\left(\bar{u}_{j} k_{t}\right) & =P+\frac{\partial}{\partial x_{j}}\left[\left(\nu+\nu_{t}\right) \frac{\partial k_{t}}{\partial x_{j}}\right]-\epsilon, \\
P & =2 \nu_{t} \bar{S}_{i j} \bar{S}_{i j}, \\
\epsilon & =C_{\epsilon} k_{t}^{1.5} \Delta^{-1},
\end{aligned}
$$

The model coefficients $C_{\epsilon}$ and $C_{k}$ are dynamically computed as part of the solution based on the Germano identity [40] with test filter $\hat{\Delta}=2 \Delta$ by the least square minimization procedure proposed by Lilly [41].

The above equations are solved by the pimpleDyMFoam solver embodied in the open-source software OpneFOAM. The cylinder is placed in the center of a circular computation domain, which has a radial extension of $20 \mathrm{D}$. The inlet boundary is specified with a uniform and constant inflow velocity $U_{\infty}$. At the outlet, the reference pressure is set to be zero. The cylinder surface is specified as noslip, which means that the flow velocity on the surface is the same with the motion velocity. For the two spanwise ends, the slip boundary condition is employed.

The computational domain is discretized with uniformly spaced grids in the azimuthal coordinate and exponentially stretched ones in the radial direction with a growing factor of 1.035. An overview of the mesh in the vicinity of the cylinder is depicted in Fig. 1(b). Since proper mesh dependency tests have been conducted for both the static and dynamic cases in our previous work [18, the same mesh resolution, i.e., $N_{\theta} \times N_{r} \times N_{z}=160 \times 160 \times 80$ is used in the current work. The timestep is set to be $\Delta=0.01$. However, there is still the need to confirm whether the mesh resolution is adequate in the case of vibration with high forcing frequency. Such results will be presented in the next section. The simulations are started from the initial condition with $U=(1,0,0)$ for the whole domain except the surface of the cylinder, where the noslip boundary condition is applied. The data, such as drag lift forces, mean flow fields, etc., are collected after at least 50 seconds to prevent the unphysical initial transients from contaminating the results. The data are recorded for more than 35 motion cycles to ensure statistical convergence. 
Table 1: Mesh dependency test for the wavy cylinder vibrating with $A_{e}=0.2$ and $f_{e}=0.3$.

\begin{tabular}{ccccccc}
\hline Case & $N_{c} \times N_{r} \times N_{z}$ & $\Delta t$ & $C_{d_{\text {mean }}}$ & $C_{l_{r m s}}$ & $f_{s}$ & $f_{e}$ \\
\hline ref [18] & $160 \times 160 \times 80$ & 0.01 & 1.307 & 1.062 & 0.168 & 0.3 \\
refined & $180 \times 180 \times 100$ & 0.005 & 1.275 & 1.057 & 0.174 & 0.3 \\
\hline
\end{tabular}

\section{Mesh dependency test}

In this section, we conduct mesh dependency test for the wavy cylinder undergoing forced vibration with $A_{e}=0.2$ and $f_{e}=0.3$. This is considered as the most critical case since the cylinder is vibrating with the highest frequency in the studied range. We compare the results between the mesh resolution used in our previous work and a refined one. The detaled mesh resolution and the corresponding results are presented in table 1. For the refined case, not only is the mesh consolidated in the three spatial directions, but also the timestep halved. In spite of this significant improvement in the spatial and temporal resolutions, the resulted drag and lift coefficients remain quite close to each other. The drag and lift coefficients are defined as

$$
\begin{aligned}
C_{d} & =\frac{F_{d}}{\rho U_{0}^{2} D_{m} H / 2}, \\
C_{l} & =\frac{F_{l}}{\rho U_{0}^{2} D_{m} H / 2},
\end{aligned}
$$

where $F_{d}$ and $F_{l}$ are the total drag and lift forces on the cylinder and $\rho$ is the density of the fluid. In addition, the time history of the lift coefficient is put to the spectral analysis and two distinctive frequencies, denoted as $f_{s}$ and $f_{e}$, are revealed. The peak at $f_{e}=0.3$ could be expected since it represents the effect of the external forcing. The existence of the other peak $f_{s}$ is of great significance to this the current work, and will be discussed in great detail in the coming sections. For the moment, it is sufficient to note that the obtained values at $f_{s}$ for the two meshes are also close to each other. In view of the above, it is reasonable to conclude that the mesh resolution we have used for the vortex-induced vibration 18 is adequate to carry out the forced vibration simulations.

\section{Results and discussion}

\subsection{The flow control of the static wavy cylinder}

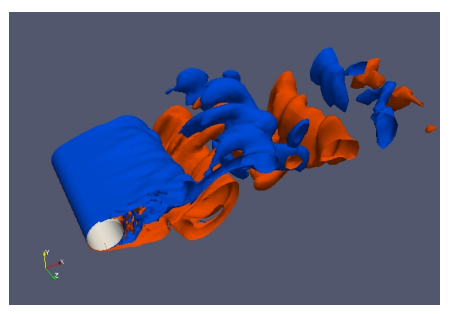

(a) normal cylinder

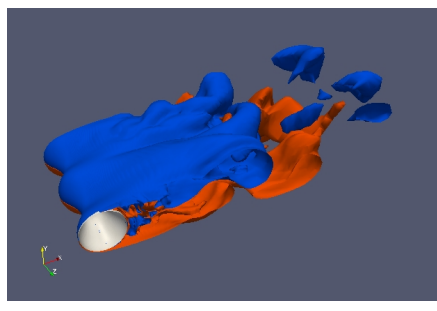

(b) wavy cylinder

Figure 2: Contour of $\omega_{z}= \pm 0.5$ of the static normal and wavy cylinders at $R e=5000$. Recreated from ref [18] with the permission from Elsevier.

The flow control efficacy of the wavy cylinder in the static configuration is revisited here to facilitate the discussion in the forced vibrations. Fig. 2 presents the snapshots of the contours of the axial vorticity $\omega_{z}= \pm 0.5$ for the normal and wavy cylinders at $R e=5000$ in the static configuration. For the normal cylinder, well-organized Kármán vortices could be clearly observed. However, in the case of the wavy cylinder, the free shear layers are greatly stabilized and roll up at far wake. The developed vortices appear to be much weakened compared with the normal cylinder. The differences in the vorticity pattern could also be quantitatively described by the spectrum of the lift coefficients, 


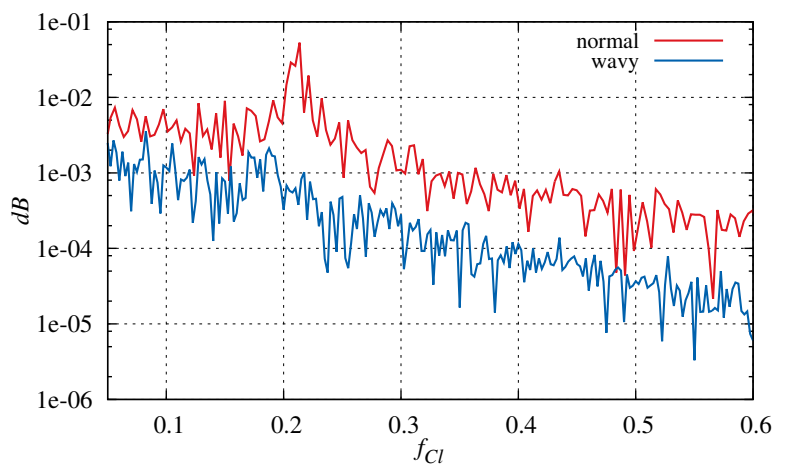

Figure 3: Frequency analysis of the lift force coefficients at fixed configuration.

as is shown in Fig. 3 A single sharp peak at $f_{v}=0.21$ representing the vortex shedding process could

be discerned for the normal cylinder, whereas such frequency is not exhibited in the case of the wavy cylinder.

Even with such strong suppression of the Kármán vortices, in the flexibly mounted case, the wavy cylinder exhibits quite large vortex-induced vibrations and the response curves in terms of both amplitude and frequency resemble that of the normal cylinder [18. In the following paper, we will present results from the perspective of forced vibration in attempt to shed some light upon the destabilization of wavy cylinder in the free vibrations.

\subsection{Global responses: comparison with the normal cylinder}

In this section, the wavy cylinder is regarded as whole and its aerodynamic responses are compared with the normal cylinder in the span-wise averaged sense. Fig. 4 presents the overall behavior of the mean drag, rms lift coefficients and the phase angle as a function of the oscillation frequency at $R e=5000$ and $A / D=0.2$. Here the phase angle $\phi$ is calculated as the phase difference between the lift coefficient and the displacement at $f_{e}$. At small forcing frequencies, the force coefficients of both the normal and wavy cylinders are kept at low values and increase slowly with $f_{e}$. The lift force is generally out-of-phase with the displacement in this regime, and the phase angle gradually decreases with increasing $f_{e}$. A sharp increase in the force coefficients, together with an abrupt decrease in the phase angle, occurs at $f_{e}=0.17$ and 0.18 for the normal and wavy cylinders, respectively. After the sudden change, $C_{d}$ gradually decreases and is kept at a value of around 1.3 for both cylinders. $C_{l}^{\prime}$, however, drops slightly and then rises again at further higher oscillation frequencies. Besides, $C_{l}$ becomes almost completely in phase with the displacement at the high $f_{e}$ regimes. Except for the small difference in the critical oscillation frequency for triggering the sudden transition, the response curves of the wavy cylinder largely resemble those of the normal cylinder.

To further explore the entanglement between the external forcing and the inherent vortex shedding, FFT analysis is applied to the time histories of the lift coefficients, and the resulted spectra are presented in Fig. 5 . For the normal cylinder, typically the lift spectra is characterized by two distinctive peaks, one at $f_{e}$, manifesting the effect of the forced oscillation, and another at $f_{s}$, which is close to, but not necessarily equals to the natural vortex shedding frequency of a fixed cylinder $f_{v}$. Depending on the amplitudes of the two peaks, the studied $f_{e}$ range could be divided into three stages. Starting from low oscillation frequency, the amplitudes of the two peaks at $f_{e}$ and $f_{s}$ are both kept at low values and are comparable with each other. Particularly, as will be discussed in more detail in the texts that follow, at the smallest forcing frequency such as $f_{e}=0.1 \sim 0.11$, the strength at $f_{s}$, which is incurred by the natural vortex shedding, exceeds that at $f_{e}$. As $f_{e}$ increases and approaches the natural shedding frequency, we enter the second stage, in which $f_{s}$ is captured by the harmonic motion and the spectra is featured by a single strong peak at $f_{e}$. This is known as the famous lock-in phenomenon that have been the focal of many related researches mentioned in section 1 . During the course of lock-in, the intensity of the single peak undergoes an abrupt jump between $f_{e}=0.16 \sim 0.17$, as has been depicted in Fig. 4. Further increasing $f_{e}$ results in the third stage, in which the Strouhal frequency revives 


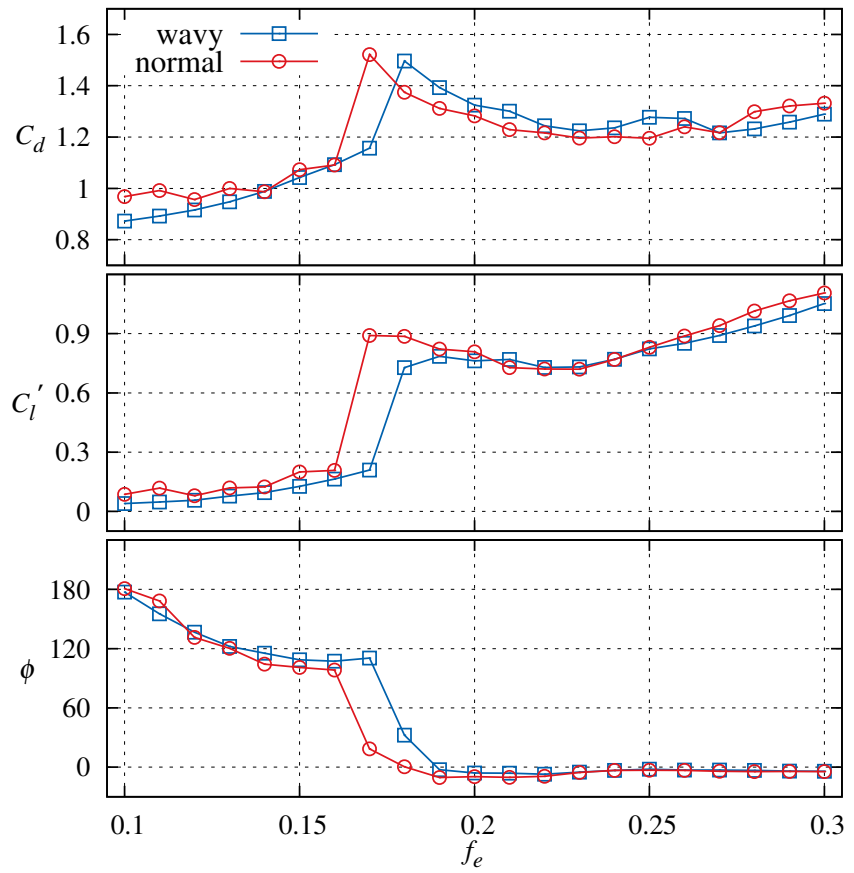

Figure 4: Variation of mean drag, rms lift coefficients and phase angle with the excitation frequency

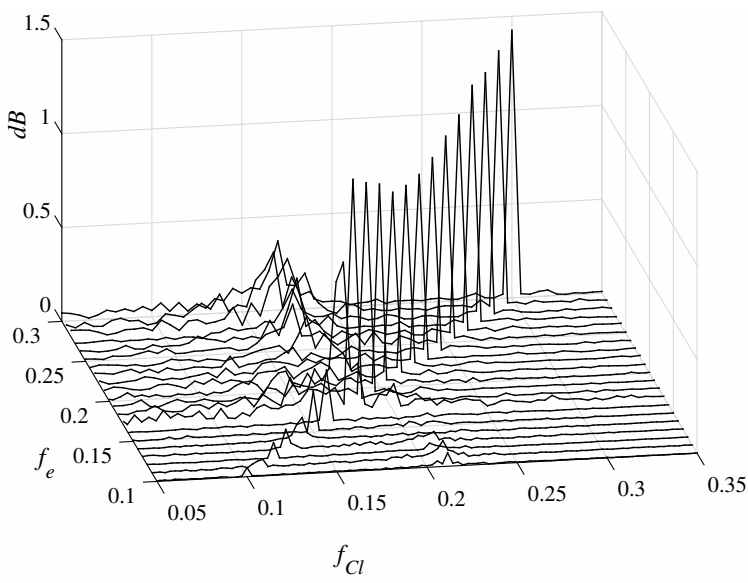

(a) normal cylinder

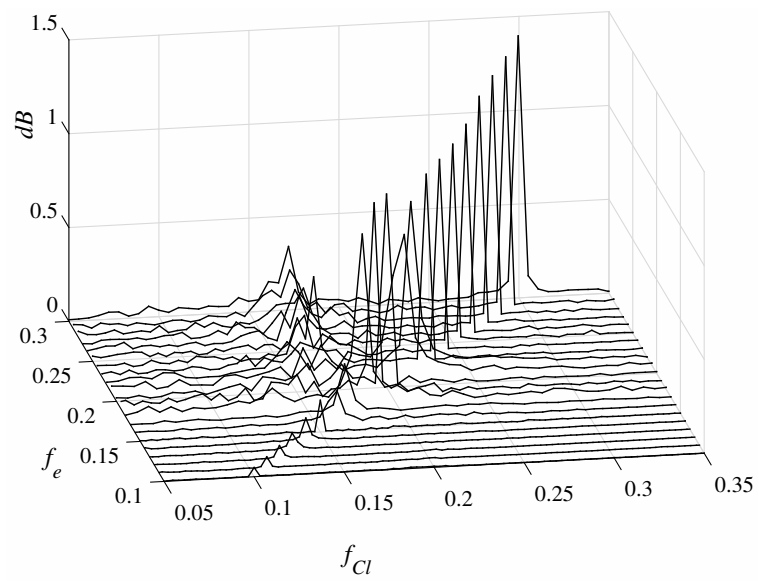

(b) wavy cylinder

Figure 5: Variation of the lift spectra with oscillation frequency $f_{e}$

at $f_{s} \approx 0.16$ and its magnitude appears stronger than that at the low forcing frequency stage. The intensity at the $f_{e}$ peak is much higher than its peer at $f_{s}$. The variation of the $f_{e}$ intensity conforms to that has been described in the overall rms lift coefficient in Fig. 4. The described features of the lift spectra is in great agreement with that of [25], in which the cylinder was studied with $A_{e}=0.25$ at $R e=4410$.

As for the wavy cylinder, although the natural shedding frequency appears to be absent in the fixed state, when forced to oscillate transversely, its spectral behavior exhibits certain similarities with that of the normal cylinder. This is especially true to the high $f_{e}$ stage, since other than the forcing frequency, a significant peak mounts up at around $f_{s} \approx 0.16$, which is reminiscent of the Strouhal frequency of the normal cylinder. Judging from Fig. 5(b) the situation appears quite different at the low forcing frequency stage, at which the spectrum seems to be dominated by a single frequency at $f_{e}$ and the component at $f_{s}$ is not observable. As a matter of fact, it will be shown in the following texts 

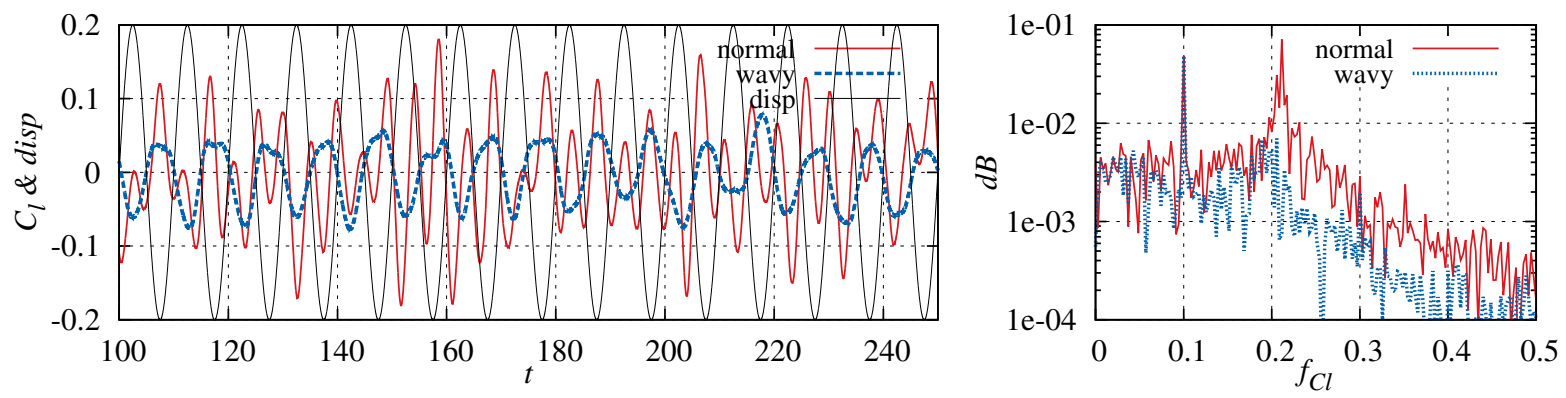

(a) $f_{e}=0.1$
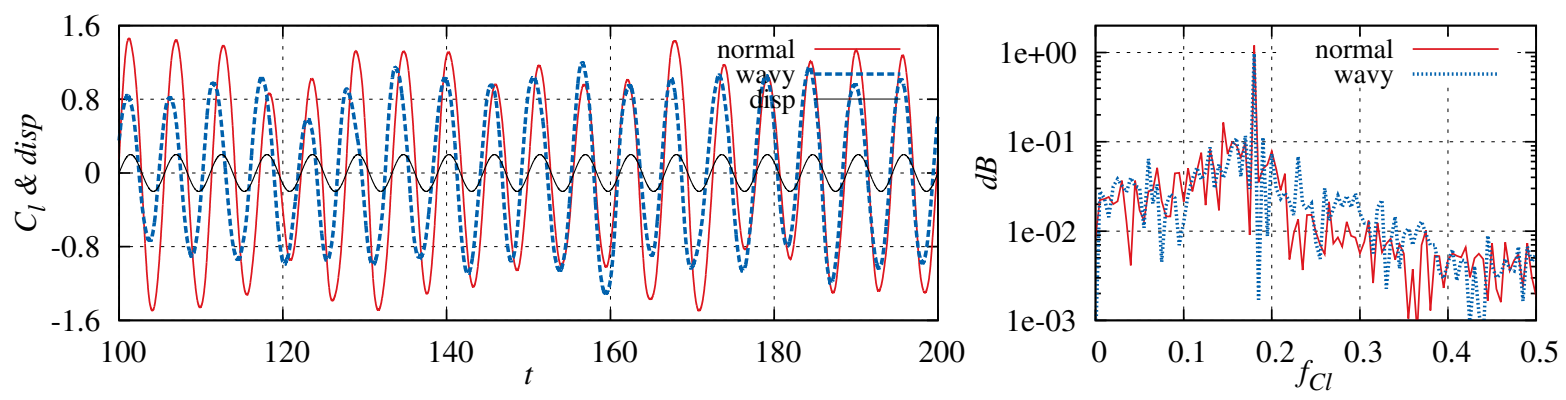

(b) $f_{e}=0.18$
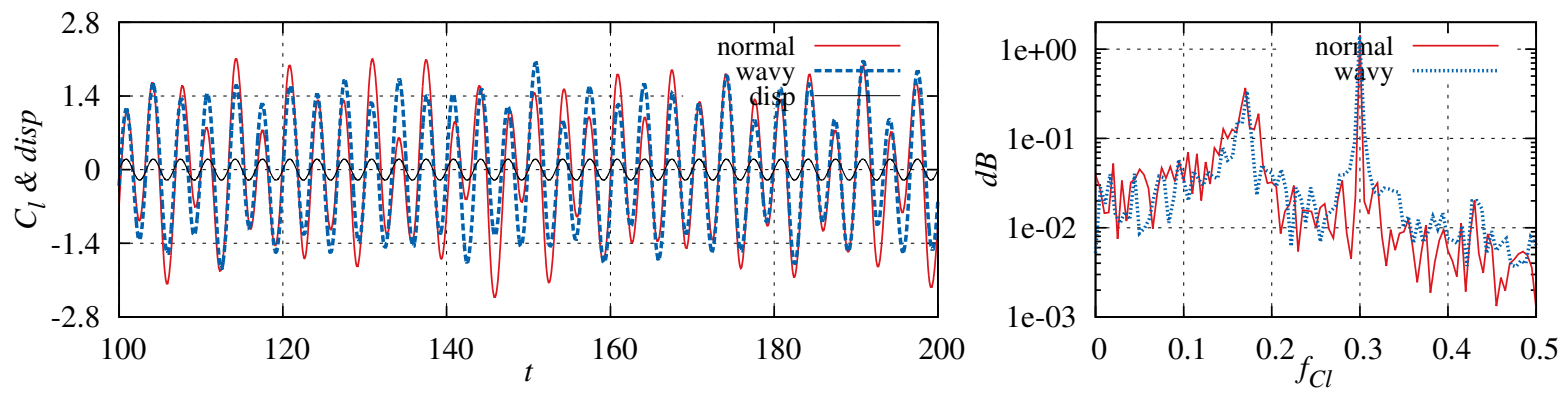

(c) $f_{e}=0.3$

Figure 6: Comparison of the time history (left) and power spectra (right) of the lift force coefficients between the normal and wavy cylinder at three typical forcing frequencies.

that the peak at $f_{s}$, small as it is, does exist in the spectrum. Similar to the normal cylinder in the lock-in regime, in between the low and high forcing stages the spectrum is dominated by a single peak at $f_{e}$. This will also be inspected more closely in the next paragraph.

Fig. 6 presents a detailed comparison of the time history and spectra of the lift force coefficients between the normal and wavy cylinders at three forcing frequencies, each of which represents the three stages described in Fig. 5. At small forcing frequency $f_{e}=0.1$, the absolute values of $C_{l}$ for both the normal and wavy cylinders are small and the former is clearly larger than the latter. The spectrum on the right side reveal the detailed information of the frequency contents. For the normal cylinder, the peak at $f_{s}$ is slightly higher than $f_{e}$, indicating that the effect of the forcing has not surpassed that of the natural vortex shedding. As for the wavy cylinder, the peak magnitude at $f_{e}$ almost coincide with the normal cylinder. Another peak, broader and much weaker, surfaces at $f_{s} \approx 0.2$, which is slightly smaller than that of the normal cylinder. Owing to the difference in the $f_{s}$ component, the time history of the normal cylinder is characterized by strong entanglement between the two frequencies, while in the case of the wavy cylinder the $f_{s}$ only have slight effect in shaping the temporal behavior of the lift coefficient. By considering the extremity of zero forcing frequency, which translates to the static configuration where full suppression of the Strouhal frequency is achieved, it could be anticipated that the intensity of the Strouhal frequency will keep deteriorating with decreasing forcing frequency. The 
lift spectra at $f_{e}=0.18$ of both cylinders are characterized by a single frequency at $f_{e}$. Note that for the static cylinder, the natural vortex shedding is 0.21 for the normal cylinder, and, judging from the $f_{s}$ peak in Fig. 6(a), 0.20 for the wavy cylinder. The case of $f_{e}=0.18$ is certainly in the range of the frequency lock-in for both the normal and wavy cylinders. It could also be observed in Fig. 6(b) that at the current forcing frequency the lift of the normal cylinder is almost in phase with the displacement, while for the wavy cylinder the lift lags behind the displacement with a small angle. When it comes to $f_{e}=0.3$, once again two distinctive peaks emerges. The second peak at $f_{e}$ is significantly higher than the $f_{s}$ for normal and wavy cylinders alike, indicating the dominance of the forced motion over the natural vortex shedding. Unlike the case at $f_{e}=0.1$, at high forcing frequency the spectra of both cylinders resemble each other at their respective peaks, resulting in the great similarity in the lift coefficients.

In the light of the discussions above, we are now in the position to discuss the possible mechanism for the destabilization of the wavy cylinder in the free vibration. In our previous paper [18, we have related the VIV of the wavy cylinder with that of a normal cylinder at $R e<47$, for which the Kármán atex shedding has yet to be triggered 42 . For the mechanism of the latter case, recent linear stability analysis 43, 44 have made it clear that while the fluid wake mode is always asleep, it is the unstable structure mode that drives the coupled fluid-structure system to the linear growth, which is then limited by the nonlinearity of the fluid and eventually arrive at the limit cycle VIV. Based on the phenomenological similarity, it was tempting to ascribe the VIV of the wavy cylinder also to the same mechanism. However, from the spectral analysis presented above, it is clearly demonstrated that the Strouhal frequency that is concealed in the fixed configuration does revive in the forced vibrations and play its role in modulating the lift coefficients, leading to similar behavior with the normal cylinder. This Strouhal frequency represents the wake mode revealed in the linear stability analysis [43, 44]. Thus, the VIV of the flexibly mounted wavy cylinder should also be rooted in typical unstable structural mode. As a matter of fact, it will be shown in section 5.4 that in the forced vibrations cases the very mechanism that suppress the vortex shedding in the fix configuration will be lessened or even removed in the forced vibrations, rendering the wavy cylinder very similar to the normal one. However, as of now it is still not clear under what mechanisms the wake mode loses its stability in the initial stages of the free vibration. Further investigations are still needed to address this issue.

\subsection{Sectional force analysis}

The discussions in the previous section have been focused on the comparison of the global responses between the normal and wavy cylinders. On the other hand, the span-wise undulation in the wavy cylinder provides a fertile ground for the discussion of the sectional features. In this section, the inner characteristics within the axial extent of the wavy cylinder will be discussed based on the sectional force coefficients, which are defined based on the slices of the sectional mesh and are calculated as

$$
\begin{aligned}
& C_{d}(z)=\frac{F_{d}(z)}{\rho U_{0}^{2} D(z) \Delta L / 2}=\sum_{0}^{N_{c}} C_{p} \cdot n_{x} \cdot \Delta \theta, \\
& C_{l}(z)=\frac{F_{l}(z)}{\rho U_{0}^{2} D(z) \Delta L / 2}=\sum_{0}^{N_{c}} C_{p} \cdot n_{y} \cdot \Delta \theta,
\end{aligned}
$$

in which $F_{d}(z)$ and $F_{l}(z)$ are the drag and lift forces on the mesh slice with width of $\Delta L$ at spanwise normal vectors.

\subsubsection{Sectional distribution of drag and lift force coefficients}

The sectional time-averaged drag and rms lift force coefficients are summarized in Fig. 7. For the drag coefficient (Fig. 7(a) , significant variation is generated in the spanwise direction. For all the forcing frequencies, the minimum $C_{d}$ is without an exception located at the middle plane in between the node and saddle planes. This is owing to the large spanwise geometric gradient $n_{z}$ consuming 

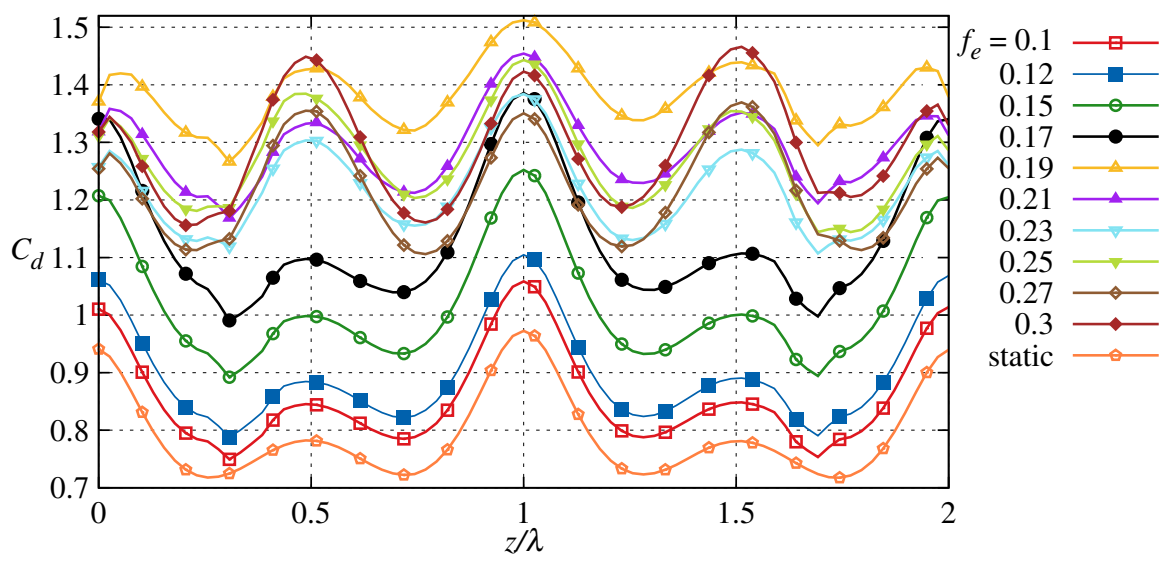

(a) The mean drag coefficients

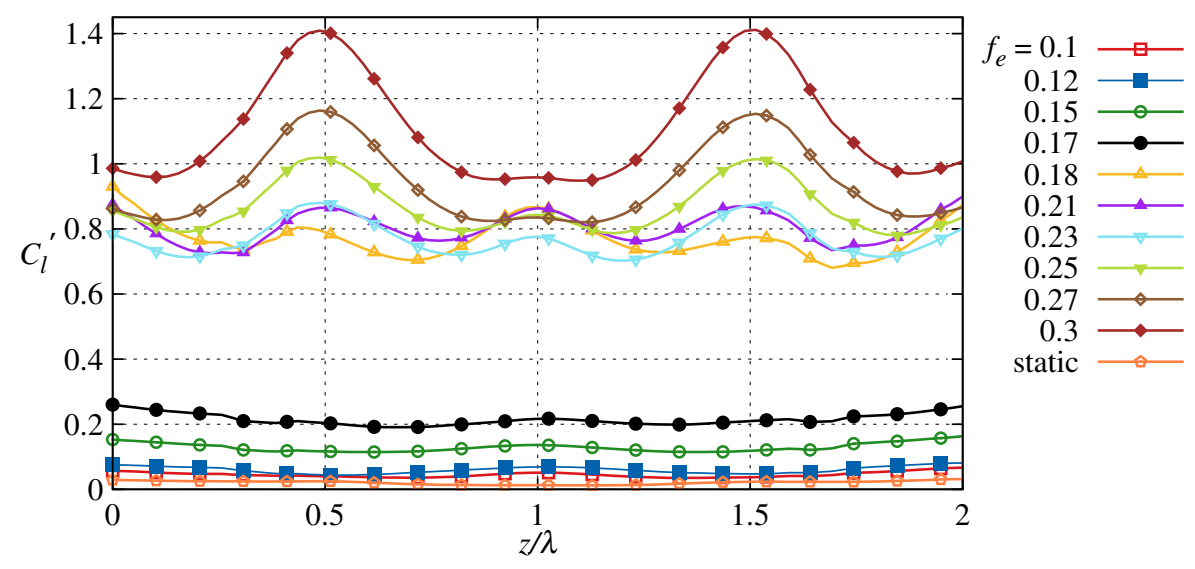

(b) The rms lift coefficients

Figure 7: Spanwise distribution of the force coefficients at selected frequencies

a large portion of the magnitude of the surface normal vector (whose magnitude is unity), resulting in much smaller $n_{x}$ and $n_{y}$, which are key ingredients in computing the sectional drag and lit force coefficients as are shown in Equations 10 and 11. Things differ for the location of the maximum drag coefficient. In the static configuration, the largest $C_{d}$ is found at the node planes. This feature persists for a large range of forcing frequencies from $f_{e}=0.1$ till $f_{e}=0.25$, even though the difference of the values at node and saddle grows smaller with the increasing forcing frequency. It is not until $f_{e}$ reaches 0.27 that the drag coefficient at the saddle begins to exceed that at the node. However, even at the highest forcing $f_{e}=0.3$, the difference between the node and saddle remains small. For all the cases studied here the curve of the sectional drag in one wavelength resembles a $\mathrm{W}$ shape.

On the other hand, the situation for the rms lift coefficient is largely divided depending on the forcing frequency. For $f_{e} \leq 0.17, C_{l}^{\prime}$ remains small and increase slowly with the forcing frequency. Along the axial direction, the spanwise variation of the lift coefficient is also small, nevertheless, it could still be discerned that in the forced cases $C_{l}^{\prime}$ reaches maximum at the node and minimum at the middle plane. The jump occurs abruptly at $f_{e}=0.18$, and the lift coefficient has been kept at high values ever since. Along with the jump, the sectional difference of $C_{l}^{\prime}$ also enlarges. At $f_{e}=0.18 \sim 0.21$, in one wavelength the $C_{l}^{\prime}$ curve features a W shape with the lift coefficient at the node slightly larger than the saddle. For further higher forcing frequencies, i.e., $f_{e}>0.23$, the lift coefficient at the saddle keeps swelling up at exceeds that at the node. The curve at one wavelength 
and lift coefficients undergo such significant changes in the studied forcing frequencies, the sectional forces, in which the local diameter $D(z)$ is incorporated, is always larger at the node and smaller at the saddle.

\subsubsection{Span-wise correlation and coherence}

The spanwise correlation of the lift coefficients measures the three dimensionality in the near wake of the body. The correlation coefficient between two signals $X(t)$ and $Y(t)$ is defined as

$$
r=\frac{\sigma_{X Y}}{\sigma_{X} \sigma_{Y}}
$$

in which $\sigma_{X Y}$ is the covariance and $\sigma_{X}$ and $\sigma_{Y}$ represent the standard deviations. In the current work, a consecutive correlation curve for the lift coefficients could be obtained by applying equation 12 to the whole extent of the cylinder with reference to one fixed end. Such results are presented in Fig. 8 for the wavy cylinder. When the wavy cylinder is static, the correlation coefficient deteriorates swiftly in the spanwise direction. Such sharp decrease in the correlation owes its origin to the completely suppressed vortex shedding. As has been elucidated in our previous work [18, the small lift coefficient on the static wavy cylinder is mainly caused by the chaotic turbulence effect and thus features low correlation. Compared with the static case, significant enhancement in the correlation could be observed for the externally excited wavy cylinders, even at the mildest forcing $f_{e}=0.1$. The correlation coefficients generally increases with the increasing forcing frequency, and this trend is not affected by whether the vortex shedding is locked to the forcing or not. The above described features are also generally true for the normal cylinder, which is omitted here for the sake of brevity. In addition, a spanwise undulated pattern, which conforms to the shape of the geometric waviness, could be noticed in the forced vibrating cases. Since in the calculation of the correlation the reference point is selected at cylinder's one end (node), the correlation always increase slightly when it approaches the node. Such phenomenon is also observed in the free vibration of the same wavy cylinder [18. This strong spatial undulation indicates clearly that the flow dynamics in the near wake is significantly affected by the cylinder motion.

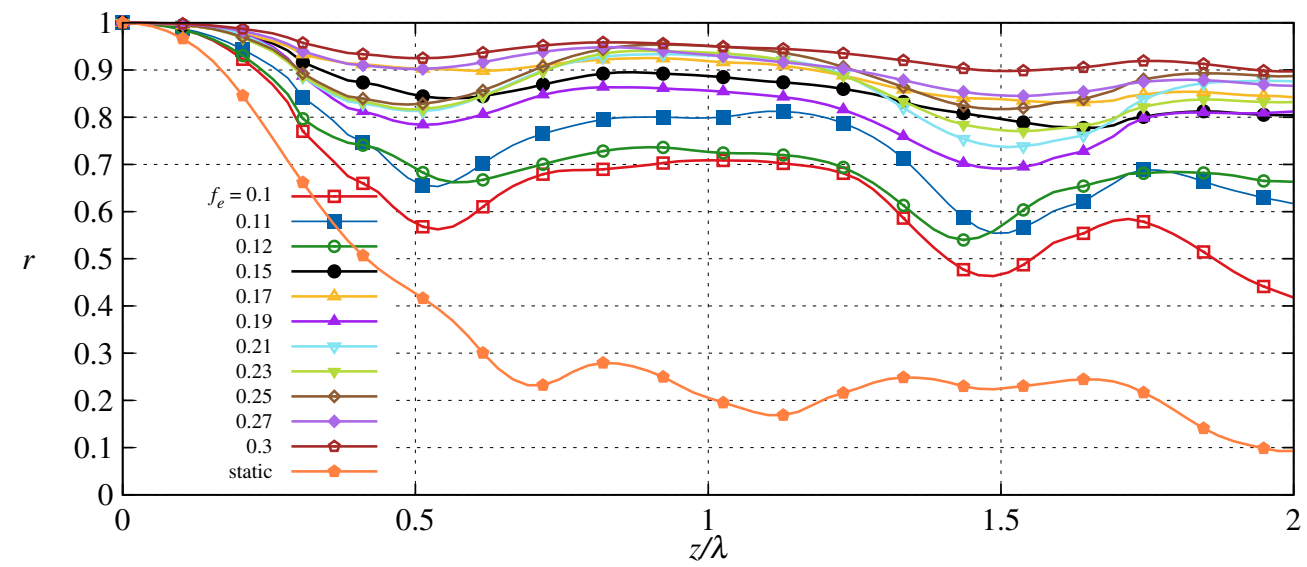

Figure 8: Span-wise correlation of the lift force coefficients

While the correlation analysis measures the similarity of the spanwise lift coefficients in the time domain, more information could be retrieved from the spectral coherence analysis, which inspects the degree of dependency of the sectional lift coefficients in the frequency space. The magnitude-square coherence is defined as

$$
h=\frac{\left|P_{X Y}(f)\right|^{2}}{P_{X}(f) P_{Y}(f)},
$$

in which $P_{X Y}$ is the cross power spectral density of the two signals and $P_{X}$ and $P_{Y}$ are the power spectrum density of the respective signals. Similar to the correlation, equation 13 is used by fixing $X$ to 


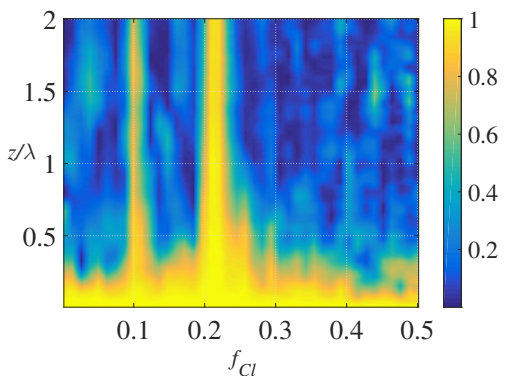

(a) $f_{e}=0.1$

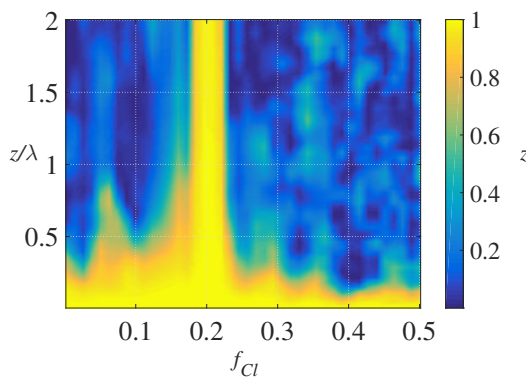

(d) $f_{e}=0.2$

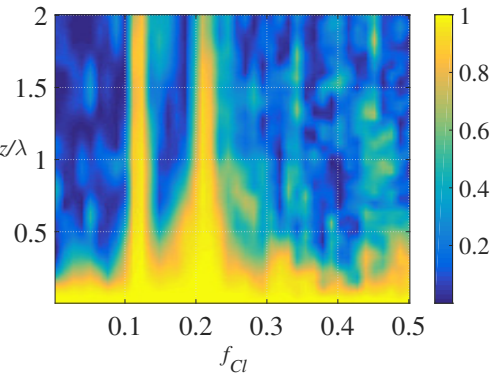

(b) $f_{e}=0.12$

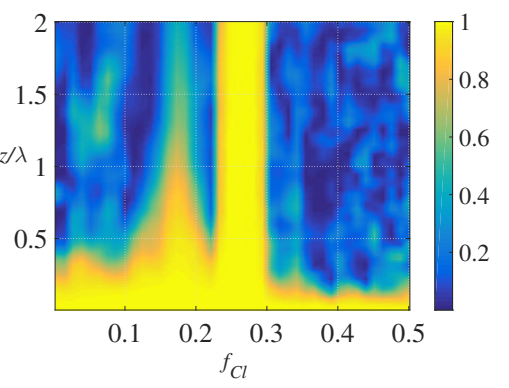

(e) $f_{e}=0.26$

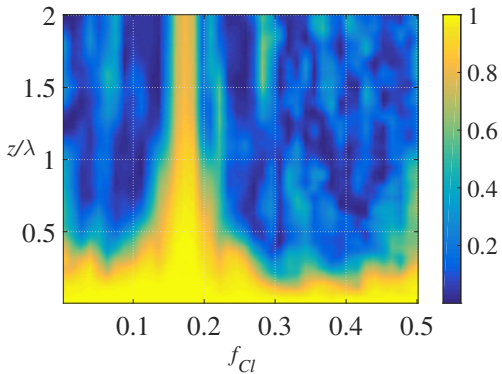

(c) $f_{e}=0.17$

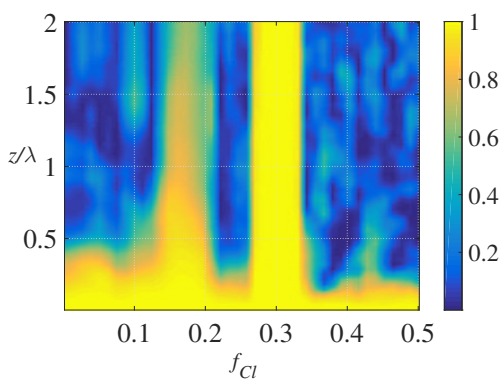

(f) $f_{e}=0.3$

Figure 9: Span-wise distribution of the magnitude-squared coherence of the lift force coefficients of the normal cylinder

$z=0$ and sweeping $Y$ all over the axial length. Selected results are presented in Fig. 9 for the normal cylinder and in Fig. 10 for the wavy cylinder. In each of the figures, the horizontal axis indicates the lift frequency $f_{C l}$ and vertical axis is the span-wise coordinate $z$ normalized by the wavelength $\lambda$. For the normal cylinder, at $f_{e}=0.1$, two distinctive frequencies are illuminated, representing the forcing and the Strouhal frequency. The coherence at $f_{C l}=0.1$ gradually weakens through the spanwise extent, while at $f_{s}$ it is better maintained. The situation gets inversed when it comes to $f_{e}=0.12$, where the coherence at the Strouhal frequency gradually weakens while that at the forcing frequency is kept high. At $f_{e}=0.17 \sim 0.2$, lock-in occurs and the two frequencies merge into one illuminated band. As the forcing frequency increases to 0.26 and 0.3 , the Strouhal frequency recurs, and its intensity of coherence becomes much weaker than its peer at $f_{e}$, which is featured by almost full spanwise coherence. Since for most of the cases spectral intensity of the lift coefficient at $f_{e}$ exceeds that at $f_{s}$, the spanwise correlation as we have discussed in the previously is also found to be positively related to the enhancement of the coherence at $f_{e}$.

The coherence spectrum of the wavy cylinder exhibits many similar features with the normal cylinder, especially at high frequencies $\left(f_{e}=0.26,0.3\right)$, where the Strouhal frequency is clearly manifested in addition to the almost fully coherent forcing frequency. At $f_{e}=0.17$ and 0.2 , the two frequencies merges into one. This serves as another solid indication for the occurrence of lock-in. Things are not exactly the same at the small forcing frequencies. At $f_{e}=0.1$ and 0.12 , while the forcing frequency is clearly illuminated in Fig. 9-10, the coherence at the Strouhal frequency is discernible but vague. This comparison corresponds well to the high and low spectral intensity at $f_{e}$ and $f_{s}$ of the wavy cylinder, as has been depicted in Fig. 6(a) In view of the above, one important conclusion that could be drawn from Fig. 10 is that during the forced vibration, the Strouhal instability does resurrect and its property appears to be very similar to that of the normal cylinder.

\subsection{Vorticity patterns}

\subsubsection{Evolution of the spanwise vorticity}

Fig. 11 presents the evolution of the vorticity dynamics for the wavy cylinder over one cycle of the motion. The contours of the spanwise vorticity $\omega_{z}$, which is of relevance to the vortex shedding phenomenon, are employed to visualize the vortices. The five snapshots in each subfigure are taken 


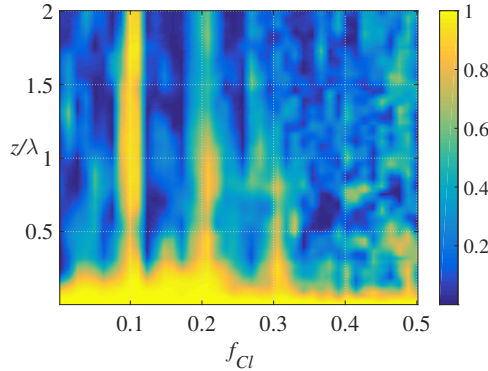

(a) $f_{e}=0.1$

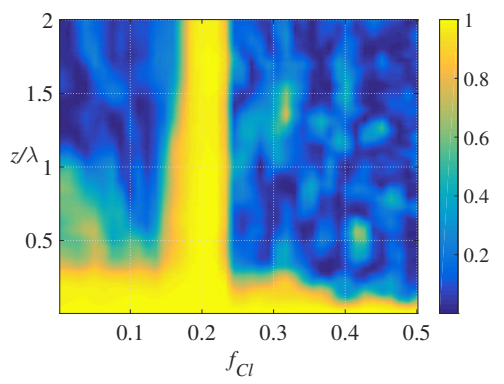

(d) $f_{e}=0.2$

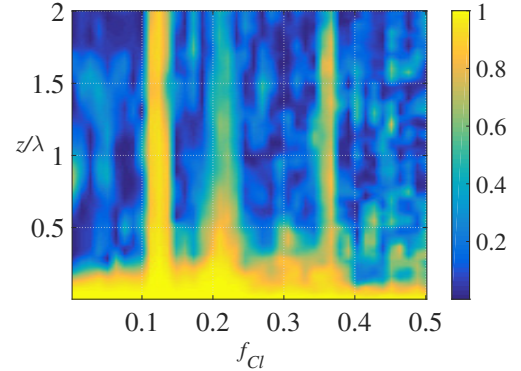

(b) $f_{e}=0.12$

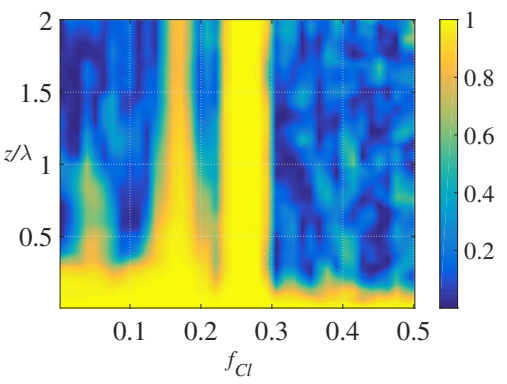

(e) $f_{e}=0.26$

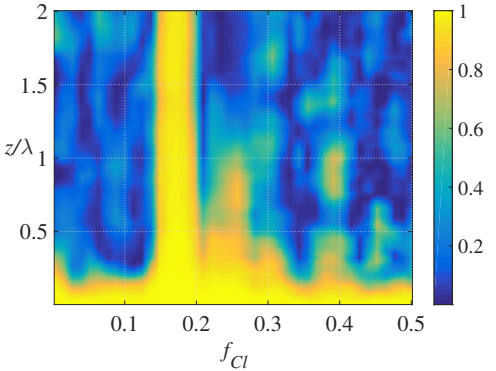

(c) $f_{e}=0.17$

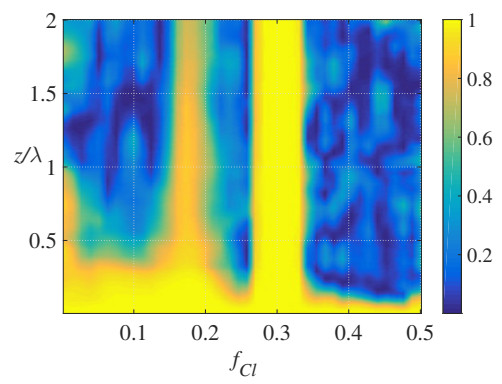

(f) $f_{e}=0.3$

Figure 10: Span-wise distribution of the magnitude-squared coherence of the lift force coefficients

at the instants when the cylinder is at the top (I), middle with downward motion (II), bottom (III), middle with upward motion (IV), and again top (V) positions. At $f_{e}=0.1$, the lift coefficient remains low and in one cycle of motion, it consists multiple frequency components. At the top position (I), the negative vorticity develops on upper side of the cylinder and detaches when the cylinder moves down to the middle position (II). Meanwhile, the upper free shear layer breeds a vortex and is ready to shed at the bottom position (III). From this perspective, the situations at the top and bottom locations very similar. As the cylinder rises again to the middle position (IV), the newly generated negative vortex sheds. Thus, at $f_{e}=0.1$ one cycle of motion corresponds to two cycles of vortex shedding, which corresponds to the peak at $f_{s} \approx 0.2$ as is observed in Fig. 6(a) However, the shedding of the vortices takes place at relatively far wake, and the effect of the natural vortex shedding on the total lift coefficient remains small.

At $f_{e}=0.17$, which is right before the abrupt jump occurs, the free shear layer with negative vorticity breeds on the upper side of the cylinder at the top position (I), and then sheds as the cylinder moves to position (II), resulting in a minimum value in the lift coefficient. For position III and IV, the same process occurs for the positive vorticity on the bottom side of the cylinder. The vorticity patterns clearly show that only one cycle of vortex shedding occurs in one cycle of the motion, indicating the that the vortex shedding takes place at a location much closer to the cylinder than that of $f_{e}=0.17$. The contraction of the wake clearly signifies the abrupt jump in the drag and lift coefficients. The upper free shear layer with negative vorticity is still in its infancy at position I and remains attached at position II, then it sheds shortly before the cylinder reaches the bottom (III).

The situation at $f_{e}=0.26$ is quite close to that at $f_{e}=0.18$, except that the lift coefficient becomes almost totally in phase with the displacement. Each time the cylinder is at the top position, positive vorticity shed at the bottom side resulting in the maximum lift coefficient and vice versa. The spanwise vortices appears quite chaotic and decay quickly as they are convected to further downstream.

\subsubsection{Time-averaged streamwise vorticity}

According to [9, 13, 14, the mechanism for the flow control of the static wavy cylinders lies in the periodic arrangement of the streamwise vorticity, which inhibits the development of the spanwise 

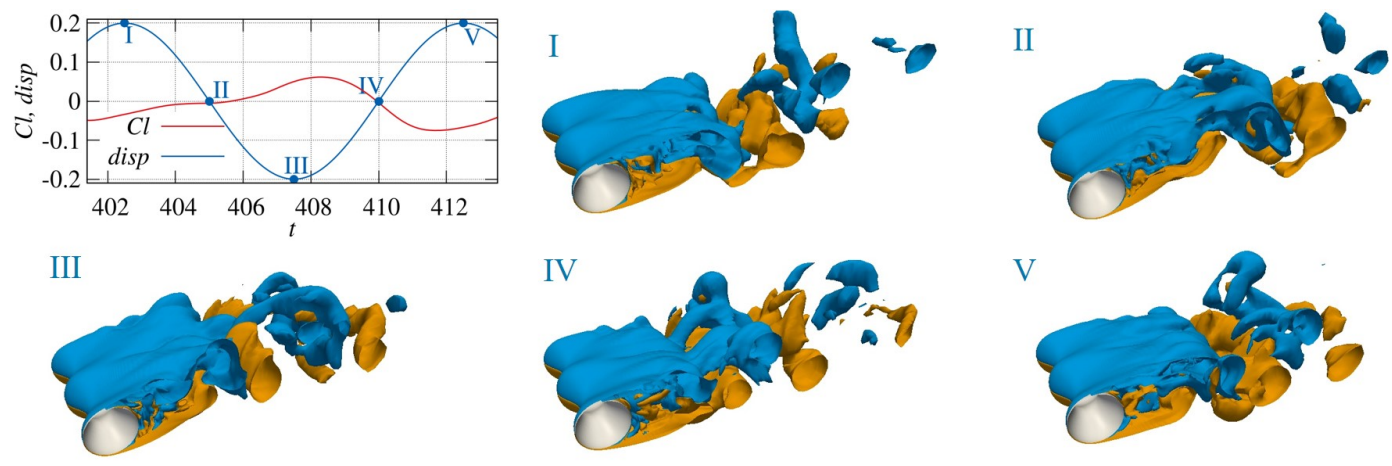

(a) $f_{e}=0.1$
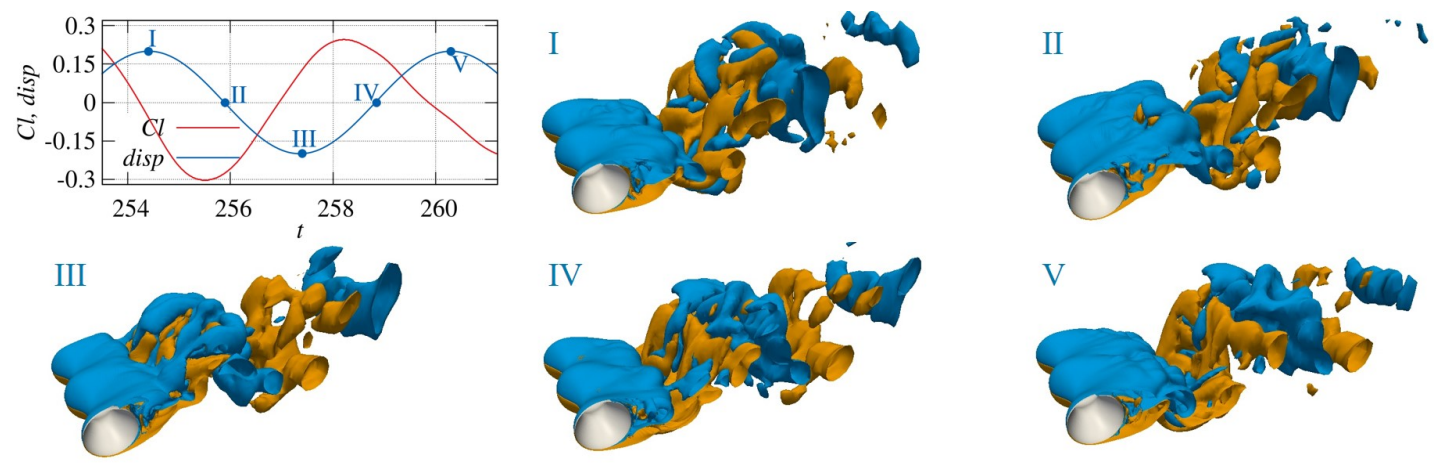

(b) $f_{e}=0.17$
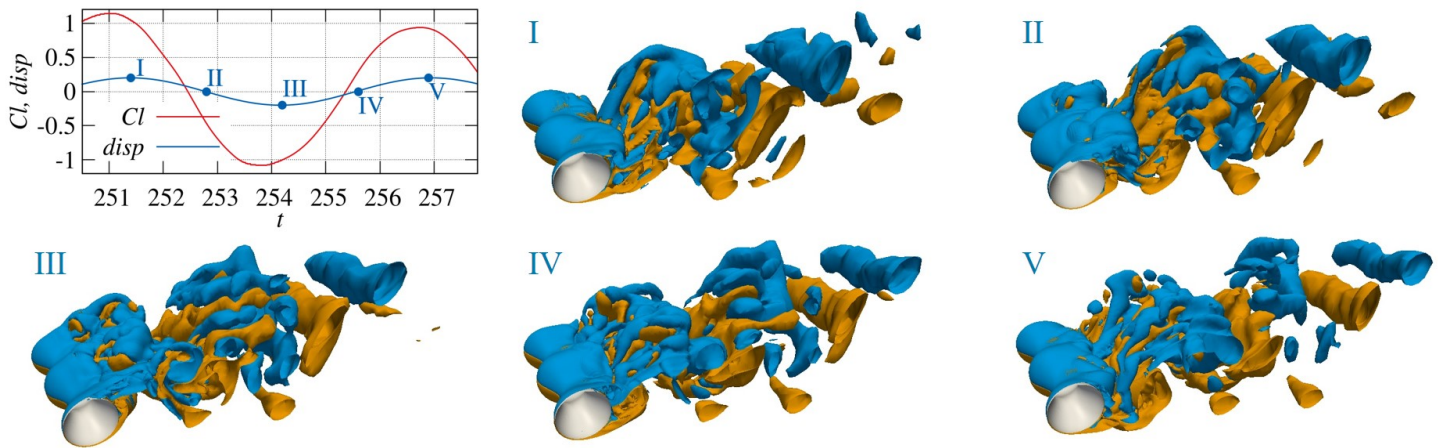

(c) $f_{e}=0.18$
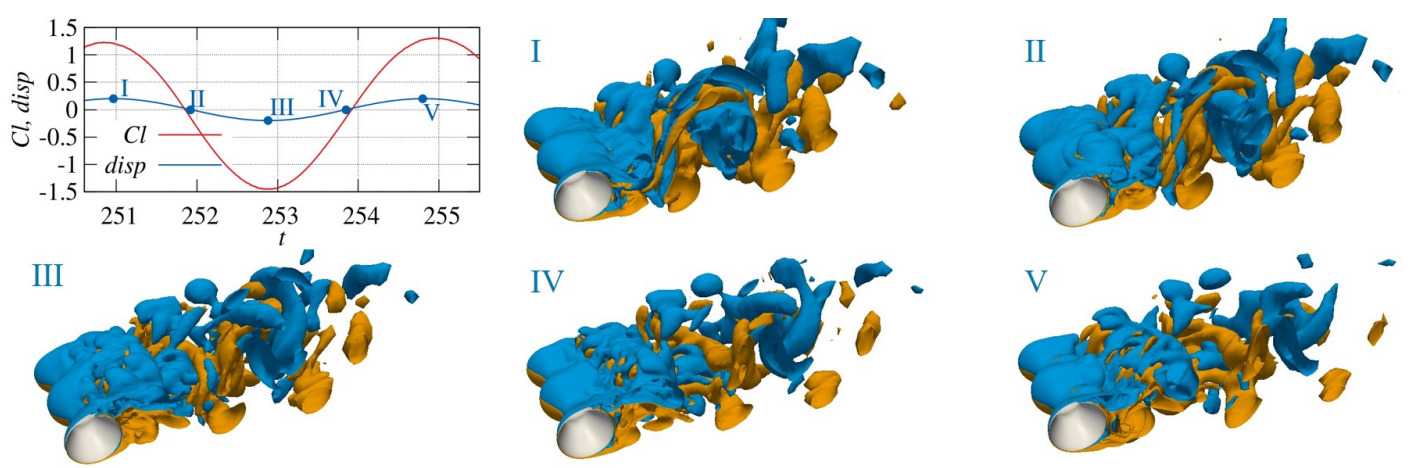

(d) $f_{e}=0.26$

Figure 11: Evolution of contour $\omega_{z}= \pm 0.5$ in the wake of the wavy cylinders. Blue and yellow indicate positive and negative spanwise vorticity. The location of the cylinder in the five snapshots are indicated in the time history plot. 
Kármán vortex shedding. This is also found to be true for the wavy cylinder studied in the current work. Fig. 12 presents the contours of the streamwise vorticity $\omega_{x}= \pm 0.5$ calculated from the timeaveraged flow filed for five cases: static, $f_{e}=0.1,0.16,0.19$ and 0.3 . In the static case, the streamwise vorticity extends far into the wake and plays its role in suppressing the formation of the spanwise Kármán vortices. At $f_{e}=0.1 \sim 0.16$, the length of the contours dwindles, indicating the lessening of the flow control efficacy. However, since $\omega_{x}$ still exists extensively in the wake, the suppression mechanism is still maintained to some extent. When it comes to $f_{e}=0.19$, significant contraction of the $\omega_{x}$ occurs, signifying the massive recession of the flow control effectiveness. At the highest forcing frequency $f_{e}=0.3$, the streamwise vorticity is suppressed so significantly that it only exists near the cylinder surface. At such an event, Kármán vortex shedding, as an inherent mechanism for the bluff bodies, no longer suffer from the obstruction from the extended $\omega_{x}$ and resurrect in the near wake. From the above discussion it is clearly manifested that the imposed cylinder motion could disrupt the formation of the streamwise vortices, which is considered as the key mechanism of the flow control in a fixed wavy cylinder. Without the existence of such a mechanism, the inherent absolute stability in the bluff body wake would recur and the wavy cylinder behaves like a normal straight cylinder in the forced vibrations.

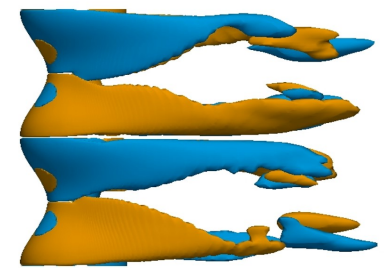

(a) static

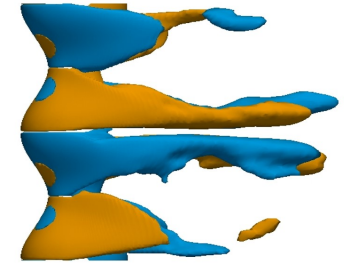

(b) $f_{e}=0.1$

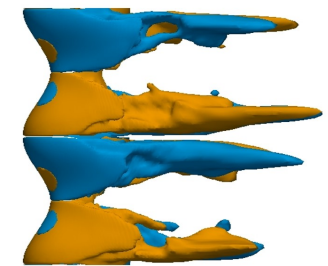

(c) $f_{e}=0.16$

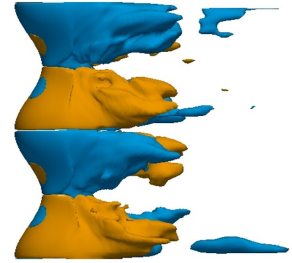

(d) $f_{e}=0.19$

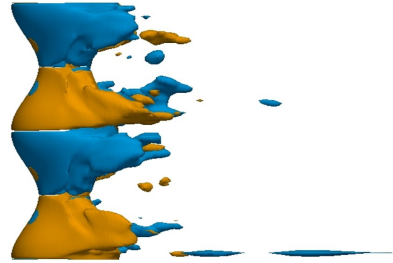

(e) $f_{e}=0.3$

Figure 12: Contours of the time-averaged streamwise vorticity $\omega_{x}= \pm 0.5$ for the wavy cylinders. Blue and yellow indicate positive and negative vorticity.

\section{Conclusion}

Intensive computational efforts have been devoted to the study of the wavy cylinder undergoing forced sinusoidal oscillation with prescribed amplitude $A_{e}=0.2$ and frequency spanning from $f_{e}=0.1$ to 0.3 at a fixed Reynolds number of 5000 . The wavy cylinder is optimally designed so that it completely suppresses the Kármán vortex shedding in the stationary configuration. Various aspects, such as the drag and lift forces, lift force spectrum, spanwise correlation and coherence, vorticity patterns, etc., has been covered. A major discovery unveiled from the current work is the resurrection of the Strouhal frequency in the forced vibrations of the wavy cylinder. This particular frequency component is also observed to submit to the forcing frequency by a typical lock-in mechanism. As a result, the response curves in terms of the force coefficients as well as the phase difference of the normal and wavy cylinders resembles each other. Detailed sectional analysis are also conducted for $C_{d}$ and $C_{l}$ in the spanwise direction. In one wavelength, while the sectional distribution of the drag coefficient generally features a $\mathrm{W}$ curve, the shape of the lift coefficients undergoes a transition from $\mathrm{W}$ to $\Lambda$. Statistical tools such as the correlation and coherence are applied to the lift coefficients to retrieve more information. The lift correlation, posing an undulation which conforms to the geometric waviness, gradually increases 
with the forcing frequency. On the other hand, the coherence spectrum clearly reveals the differences in the spanwise correlations between different frequency peers.

The discussion presented above suggests that rather than merely the destabilization of the structural mode, the free vibration of the flexibly mounted wavy cylinder [18 should be a result of the typical wake-structure interaction, which is similar to the case of the normal cylinder. Future researches are needed to understand the detailed mechanism for the revival of the Strouhal instability in the vibrating cases, especially in the initial development of the free vibration.

[11] K. Lam, F. Wang, R. So, Three-dimensional nature of vortices in the near wake of a wavy cylinder, Journal of Fluids and Structures 19 (6) (2004) 815-833.

[12] C.-Y. Xu, L.-W. Chen, X.-Y. Lu, Large-eddy simulation of the compressible flow past a wavy cylinder, Journal of Fluid Mechanics 665 (2010) 238-273.

[13] Y. Lin, H. Bai, M. M. Alam, W. Zhang, K. Lam, Effects of large spanwise wavelength on the wake of a sinusoidal wavy cylinder, Journal of Fluids and Structures 61 (2016) 392-409.

[14] Y. Hwang, J. Kim, H. Choi, Stabilization of absolute instability in spanwise wavy two-dimensional wakes, Journal of Fluid Mechanics 727 (2013) 346-378. 
[15] F. E. Fish, J. M. Battle, Hydrodynamic design of the humpback whale flipper, Journal of Morphology 225 (1) (1995) 51-60.

[16] D. Miklosovic, M. Murray, L. Howle, F. Fish, Leading-edge tubercles delay stall on humpback whale (megaptera novaeangliae) flippers, Physics of fluids 16 (5) (2004) L39-L42.

[17] K. L. Hansen, R. M. Kelso, B. B. Dally, Performance variations of leading-edge tubercles for distinct airfoil profiles, AIAA journal 49 (1) (2011) 185.

[18] K. Zhang, H. Katsuchi, D. Zhou, H. Yamada, T. Zhang, Z. Han, Numerical simulation of vortex induced vibrations of a flexibly mounted wavy cylinder at subcritical reynolds number, Ocean Engineering 133 (2017) 170-181.

[19] S. Dong, G. Triantafyllou, G. Karniadakis, Elimination of vortex streets in bluff-body flows, Physical Review Letters 100 (20) (2008) 204501.

[20] R. Bishop, A. Hassan, The lift and drag forces on a circular cylinder oscillating in a flowing fluid, in: Proceedings of the Royal Society of London A: Mathematical, Physical and Engineering Sciences, Vol. 277, The Royal Society, 1964, pp. 51-75.

[21] G. Koopmann, The vortex wakes of vibrating cylinders at low reynolds numbers, Journal of Fluid Mechanics 28 (3) (1967) 501-512.

[22] A. Ongoren, D. Rockwell, Flow structure from an oscillating cylinder part 1. mechanisms of phase shift and recovery in the near wake, Journal of Fluid Mechanics 191 (1988) 197-223.

[23] S. Krishnamoorthy, S. Price, M. Paidoussis, Cross-flow past an oscillating circular cylinder: Synchronization phenomena in the near wake, Journal of Fluids and Structures 15 (7) (2001) 955-980.

[24] J. Carberry, J. Sheridan, D. Rockwell, Forces and wake modes of an oscillating cylinder, Journal of Fluids and Structures 15 (3-4) (2001) 523-532.

[25] J. Carberry, J. Sheridan, D. Rockwell, Controlled oscillations of a cylinder: forces and wake modes, Journal of Fluid Mechanics 538 (2005) 31-69.

[26] T. Morse, C. Williamson, Prediction of vortex-induced vibration response by employing controlled motion, Journal of Fluid Mechanics 634 (2009) 5-39.

[27] H. M. Blackburn, R. D. Henderson, A study of two-dimensional flow past an oscillating cylinder, Journal of Fluid Mechanics 385 (1999) 255-286.

[28] S. Dong, G. E. Karniadakis, Dns of flow past a stationary and oscillating cylinder at, Journal of Fluids and Structures 20 (4) (2005) 519-531.

[29] P. Anagnostopoulos, Numerical study of the flow past a cylinder excited transversely to the incident stream. part 1: Lock-in zone, hydrodynamic forces and wake geometry, Journal of Fluids and Structures 14 (6) (2000) 819-851.

[30] S. Kim, P. A. Wilson, Z.-M. Chen, Numerical simulation of force and wake mode of an oscillating cylinder, Journal of Fluids and Structures 44 (2014) 216-225.

[31] S. Kumar, Navrose, S. Mittal, Lock-in in forced vibration of a circular cylinder, Physics of Fluids 28 (11) (2016) 113605.

[32] C. Williamson, A. Roshko, Vortex formation in the wake of an oscillating cylinder, Journal of Fluids and Structures 2 (4) (1988) 355-381.

[33] T. Sarpkaya, A critical review of the intrinsic nature of vortex-induced vibrations, Journal of Fluids and Structures 19 (4) (2004) 389-447. 
495

[34] C. Williamson, R. Govardhan, Vortex-induced vibrations, Annual Review of Fluid Mechanics 36 (2004) 413-455.

[35] R. Gabbai, H. Benaroya, An overview of modeling and experiments of vortex-induced vibration of circular cylinders, Journal of Sound and Vibration 282 (3) (2005) 575-616.

[36] T. Staubli, Calculation of the vibration of an elastically mounted cylinder using experimental data from forced oscillation, ASME Journal of Fluids Engineering 105 (2) (1983) 225-229.

[37] P. M. Munday, K. Taira, On the lock-on of vortex shedding to oscillatory actuation around a circular cylinder, Physics of Fluids 25 (1) (2013) 013601.

[38] J.-Z. Wu, X.-Y. Lu, A. G. Denny, M. Fan, J.-M. Wu, Post-stall flow control on an airfoil by local unsteady forcing, Journal of Fluid Mechanics 371 (1998) 21-58.

[39] C.-A. Yeh, P. M. Munday, K. Taira, Laminar free shear layer modification using localized periodic heating, Journal of Fluid Mechanics 822 (2017) 561-589.

[40] M. Germano, U. Piomelli, P. Moin, W. H. Cabot, A dynamic subgrid-scale eddy viscosity model, Physics of Fluids A: Fluid Dynamics (1989-1993) 3 (7) (1991) 1760-1765.

[41] D. K. Lilly, A proposed modification of the germano subgrid-scale closure method, Physics of Fluids A: Fluid Dynamics (1989-1993) 4 (3) (1992) 633-635.

[42] S. Mittal, S. Singh, Vortex-induced vibrations at subcritical re, Journal of Fluid Mechanics 534 (2005) 185-194.

[43] W. Zhang, X. Li, Z. Ye, Y. Jiang, Mechanism of frequency lock-in in vortex-induced vibrations at low reynolds numbers, Journal of Fluid Mechanics 783 (2015) 72-102.

[44] S. Mittal, et al., Lock-in in vortex-induced vibration, Journal of Fluid Mechanics 794 (2016) $565-594$. 